\title{
Departamento de Clínica Médica, 2002
}

\author{
Ricardo Brandt de Oliveira \\ Docente. Departamento de Clínica Médica. Faculdade de Medicina de Ribeirão Preto - USP.
}

\section{RECURSOS HUMANOS}

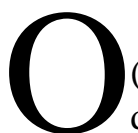

Departamento de Clínica Médica (DCM) da FMRP-USP conta com 49 docentes $(18,8 \%$ do total dos docentes da unidade), 47 dos quais em RDIDP. Todos os atuais docentes têm o título de doutor, 14 são livredocentes e 10, professores titulares. O mais jovem tem 33 anos e o mais velho 69, sendo a idade mediana de 50 anos. Conta, também, com 2 Docentes Colaboradores, ambos com doutoramento e estágio de pósdoutoramento no Exterior, contratados pela Fundação de Apoio ao Ensino, Pesquisa e Assistência do HCFMRP-USP (FAEPA).

Para vários fins, os docentes do DCM se congregam em grupos, de acordo com as suas especialidades, constituindo as Divisões do Departamento

\begin{tabular}{l} 
Quadro I - Divisóes do Departamento de Clínica \\
Médica \\
\hline Cardiologia \\
Endocrinologia e Metabolismo \\
Hematologia \\
Gastrenterologia \\
Geral e Geriatria \\
Imunologia Clínica \\
Moléstias Infecciosas \\
Nefrologia \\
Nutriçẫo \\
Pneumologia \\
Radiologia
\end{tabular}

(Quadro I). Nesse conjunto, figuram as especialidades clínicas clássicas mais a Dermatologia e a Radiologia.

O DCM conta com uma equipe de cerca de uma centena de médicos assistentes, formalmente ligados ao Hospital das Clínicas da FMRP-USP (HCRP), que cumprem funções assistenciais junto às diversas Divisões do Departamento. Embora a titulação acadêmica não seja um pré-requisito para a função, uma proporção elevada dos médicos-assistentes é constituída por mestres e doutores, muitos deles participando dos programas regulares de ensino e de projetos de pesquisa.

$\mathrm{O}$ apoio administrativo para as atividades acadêmicas, é fornecido pela secretaria do DCM que conta com 3 secretárias e 2 escriturárias da USP e 1 escriturária do HCRP. O DCM, conta ainda com 31 funcionários do HCRP (1 Chefe de Seção, 2 Encarregados de Setor e 28 Oficiais Administrativos) distribuídos entre as diferentes divisões, de acordo com o volume de atividade assistencial realizada e com 3 funcionários mantidos pela FAEPA.

$\mathrm{O}$ suporte técnico às atividades desenvolvidas nos laboratórios e serviços do DCM é dado por 25 funcionários da USP (15 Especialistas em Laboratório, 1 Biologista, 6 Técnicos de Laboratório, 1 Auxiliar de Laboratório e 2 Auxiliares de Serviços Gerais) e 91 funcionários do HCRP (43 Técnicos de Laboratório, 38 técnicos de raio X e 10 Biologistas). O DCM conta, ainda, com 1 Técnico em Informática, funcionário da USP, e 1 Técnico de Trabalho Científico, contratado pela FAEPA e 2 Especialistas em Laboratório contratados pelo programa CEPID, coordenado por docentes da Divisão de Hematologia.

Finalmente, para os cuidados de pacientes sob a sua responsabilidade, o DCM conta com uma equi- 
pe de profissionais de enfermagem do HCRP (Enfermeiros, Auxiliares de Enfermagem e Técnicos de Enfermagem), nas enfermarias, serviços e áreas de atendimento ambulatorial.

Por ocasião de sua instalação, em abril de 1954, o corpo docente do Departamento de Clinica Médica era constituído por 7 docentes, médicos formados entre 1940 e 1951, com experiência profissional prévia nos serviços do Hospital das Clínicas de São Paulo. Nenhum tinha mais que 38 anos de idade, 1 deles, o chefe, era livre-docente pela FMUSP, 1 outro tinha o título de doutor pela mesma instituição e todos eram contratados em regime de tempo integral. Durante os primeiros 2 anos, esse núcleo inicial exerceu atividades clínicas e didáticas nos 25 leitos da Enfermaria de Medicina de Homens da Santa Casa de Misericórdia de Ribeirão Preto e se dedicou a adaptar, para os fins de um laboratório de pesquisa, um pavilhão da $\mathrm{Fa}$ culdade de Medicina, recém-instalada na Fazenda Monte Alegre.

\section{ENSINO}

\subsection{Ensino de Graduação}

Como um dos grandes departamentos da FMRP, o DCM tem participação destacada nas atividades de ensino de graduação da unidade. Dezesseis disciplinas obrigatórias do curso de graduação de Medicina da FMRP são da responsabilidade de docentes do DCM (Quadro 2). Além disso, vários docentes do DCM têm participação prevista nos novos cursos recém-instalados (Fisioterapia e Terapia Ocupacional) ou em vias de instalação (Nutrição e Informática Médica).

A agenda de implantação do curso médico da FMRP determinou o momento da criação do DCM: em abril de 1954, o único encargo de seus docentes era ministrar a disciplina de Propedêtica Médica para a primeira turma de médicos da FMRP.
Em que pesem o volume e a complexidade das demandas, sempre crescentes com o passar dos anos, a recair sobre os seus docentes, o forte compromisso com o ensino de graduação do DCM não se esvaiu. Os fatos a seguir são ilustrativos.

O DCM tem, há vários anos, uma Comissão de Ensino de Graduação, de 4 membros, com mandatos definidos, com atividade permanente e que efetivamente coordena as atividades de graduação do departamento.

Apenas uma das disciplinas obrigatórias, a de Semiologia Geral, mobiliza mais de 2 dezenas de docentes do DCM durante várias semanas, a cada ano.

Em 2002, 15 disciplinas opcionais de graduação foram oferecidas por docentes do DCM.

Desde 1996, a presidência da Comissão de Graduação da FMRP é ocupada, em mandatos sucessivos, por docentes do DCM.

Nove das 45 turmas formadas na FMRP tiveram docentes do Departamento de Clinica Médica como paraninfos ou patronos.

\subsection{Ensino de Pós-Graduação}

Fortemente vinculado ao DCM, existe o programa de pós-graduação stricto sensu, denominado Clínica Médica, que conta com 47 orientadores credenciados, 40 dos quais são docentes do DCM. Desde 1973 até o final de janeiro de 2003, o programa conferiu títulos a 139 doutores e a 189 mestres. O número de titulações cresceu ao longo do tempo, o que é ilustrado pelos dados do Quadro 3, onde são comparados os números de mestrados e doutorados no triênio 1982-84, quando o programa já se encontrava plenamente implantado, com os do triênio 2000-2002.

Até o ano de 1999, o programa contava com uma área de concentração, também chamada Clínica Médica. No ano de 2000, foi criada, dentro do programa, uma nova área de concentração, Biociências Aplicadas à Clínica Médica, aberta a profissionais não médicos, na qual já foram defendidos 07 mestrados.

\begin{tabular}{|c|c|c|c|}
\hline \multicolumn{4}{|c|}{$\begin{array}{l}\text { Quadro } 2 \text { - Disciplinas do curso de graduaçăo em Medicina da FMRP sob a responsabilidade } \\
\text { do Departamento de Clínica Médica }\end{array}$} \\
\hline Semiologia Geral & Hematologia & Sistema Respiratório & Sistema Endócrino \\
\hline Sistema Cardiovascular & Sistema Digestivo & Moléstias Infecciosas & Imunologia Clínica \\
\hline Dermatologia & Nutrologia & Estágio Clínica Médica II & Estágio Clínica Médica I \\
\hline $\begin{array}{c}\text { Fundamentos } \\
\text { Humanísticos do Saber } \\
\text { Médico }\end{array}$ & $\begin{array}{l}\text { Afecçỗes do } \\
\text { Sistema } \\
\text { Genitourinário }\end{array}$ & $\begin{array}{c}\text { Introduçẫo a } \\
\text { Conhecimento da } \\
\text { Infecçẫo Hospitalar }\end{array}$ & $\begin{array}{l}\text { Ciências da Imageme } \\
\text { Física médica }\end{array}$ \\
\hline
\end{tabular}


Como demonstra o Quadro 4, nos últimos anos, ocorreu expressivo aumento de ingressos no programa, em grande parte devido à abertura da nova área de concentração. Em conseqüência, o número de matrículas ativas cresceu de 67, em 1997, para 144, em 2002.

A área de concentração Clínica Médica, criada em 1971, foi uma das pioneiras da pós-graduação na área médica no país, e obteve classificação 5 nas avaliações mais recentes, realizadas pela CAPES.

Essa área de concentração é responsável pela formação de 40 dos 49 docentes do DCM, bem como por um número expressivo de professores de escolas médicas de todas as regiões do País. Muitos egressos do programa apresentam desempenho destacado no meio acadêmico. À guisa de exemplo, a Diretoria da Faculdade de Ciências Médicas da UNICAMP foi, por duas vezes, ocupada por ex-alunos do programa, e vários egressos do programa são membros dos comitês de avaliação de pós-graduação na área médica da CAPES; no caso da Medicina I, 5 dos 16 membros o eram no biênio 2000-2001.

\subsection{Residência Médica (RM), Aprimoramento e Especialização}

Cada uma das Divisões do DCM mantém um programa de RM no HCRP. Além desses 12 programas, há o de Clínica Médica, cuja responsabilidade é compartilhada por todos os docentes do Departamento de Clinica Médica, e que constitui pré-requisito para os demais, exceto para os de Doenças Infecto-Parasitárias e de Radiologia (Quadro 5). Em 2002, os médicos residentes eram 139 (32 R1, 53 R2, 49 R3 e 5

Quadro 3 - Defesas de mestrado e doutorado no programa Clínica Médica da FMRP.USP nos triênios de 1982-1984 e 2000-2002.

\begin{tabular}{|lcc|}
\hline & $1982-1984$ & $2000-2002$ \\
\hline Mestrados & 15 & 27 \\
\hline Doutorados & 12 & 27 \\
\hline
\end{tabular}

\begin{tabular}{|lcc|}
\hline $\begin{array}{l}\text { Quadro } 4 \text { - Ingressantes no programa Clínica } \\
\text { Médica da FMRP-USP, nos triênios } \\
\text { 2000-2002 }\end{array}$ \\
\hline & $1997-1999$ e \\
\hline Mestrados & 34 & $9000-2002$ \\
\hline Doutorados & 18 & 39 \\
\hline
\end{tabular}

R4), tendo sido emitidos, no final desse ano, 53 certificados de conclusão dos diferentes programas vinculados ao DCM.

A Divisão de Nutrição é responsável por um programa de Aprimoramento em Nutrição Clínica, para nutricionistas, com duração de 2 anos, com 4 vagas, e um curso de Especialização em Nutrição, com 10 vagas.

As características do elenco de médicosresidentes vinculados ao DCM (Figura 1) retratam a evolução da prática da Medicina no âmbito do departamento.

Até o final da década de 60, a RM em clínica médica era opção de poucos, embora a sua procura viesse crescendo. Por essa época, embora o DCM já contasse com especialistas, os seus serviços especializados eram incipientes. O programa oferecido era de treinamento por 1 ano em Clínica Médica, mas não havendo definições rígidas, esporadicamente esse treinamento se estendia por tempo mais longo.

Na década de 70, a procura por 1 ano de RM em clínica médica cresceu, a ponto de obrigar o DCM a limitar vagas, e a extensão do treinamento para um $2^{\circ}$ ano passou a ser a regra, em geral, em uma das especialidades clínicas que, naquela época, ganhavam força dentro do DCM.

A partir de 1980, com o credenciamento junto à Comissão Nacional de Residência Médica, os programas de especialidade passaram a ter obriga-

\begin{tabular}{l} 
Quadro 5 - Prodramas de Residência Médica \\
vinculados ao Departamento de Clínica Médica \\
\hline Clínica Médica \\
\hline Cardiologia \\
\hline Dermatologia \\
\hline Doenças Infecciosas e Parasitárias \\
\hline Endocrinologial Metabologia \\
\hline Pneumologia \\
\hline Radiologia \\
\hline Gastroenterologia \\
\hline Geriatria \\
\hline Hematologial Hemoterapia \\
\hline Nefrologia \\
\hline Reumatologia \\
\hline Nutrologia \\
\hline
\end{tabular}




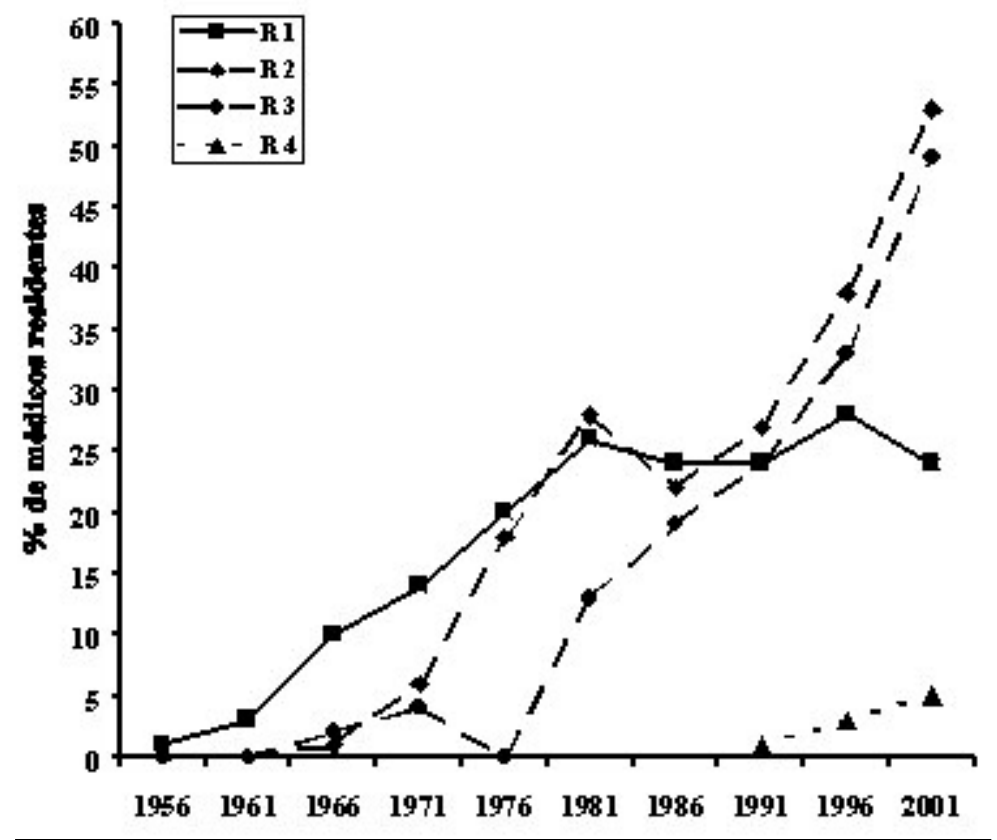

Figura 1: Evolução do quadro de médicos - residentes do Departamento de Clínica Médica entre1956 e 2001.

toriamente 1 ano de clínica médica como pré-requisito e 2 anos de duração com exceção do programa de Clínica Médica, com apenas 1 ano (o que explica o paralelismo e não superposição, das curvas de números de $R_{2}$ e $R_{3}$ a partir de então). Desde essa época, a procura pela RM foi sempre superior à oferta, de sorte que as mudanças da $R M$ do DCM foram ditadas apenas por modificações do volume e da natureza da Medicina praticada. Nota-se que o número de $R_{1}$ permanece estável nos últimos 20 anos enquanto os de $R_{2}$ e $R_{3}$ aumentaram expressiva e continuamente, refletindo os incrementos de volume e complexidade da atividade médica no âmbito do DCM. Esse aumento de complexidade é indicado, ainda, pela emergência, durante a última década, da figura do $R_{4}$ em áreas de superespecialidades.

\section{ATIVIDADES ASSISTENCIAIS}

Como decorrência natural de sua missão acadêmica, de ensino e pesquisa na área médica, o DCM mantém vínculos com instituições prestadoras de serviços de saúde. O mais antigo e robusto desses vínculos se faz com o Hospital das Clínicas da FMRP, onde se situa, atualmente, a sua sede principal. O DCM assume integralmente a responsabilidade pelo Serviço de Clínica Médica do HCRP, bem como por diversos dos seus laboratórios e serviços de apoio. Docentes do DCM exercem atividades administrativas no He- mocentro de Ribeirão Preto. Os dados apresentados a seguir dão idéia do volume dessa atividade.

No HC-Campus, o DCM dispõe de 135 leitos hospitalares, distribuídos entre as diferentes divisões, que ocupam as enfermarias do $5^{0}$ e do $6^{0}$ pavimentos do hospital, além de 5 leitos da Unidade Coronariana, no $2^{0}$ pavimento, próximo à sede da Divisão de Cardiologia, e 22 leitos da Unidade Especial de Terapia de Doenças Infecciosas (UETDI). Durante o ano de 2001, foram realizadas 4296 internações nesse conjunto de leitos. Na Unidade de Emergência, o DCM conta com 36 leitos, nos quais foram feitas 3745 internações ao longo de 2001.

Cada Divisão do DCM, com exceção da Radiologia, mantém, pelo menos, um período (manhã ou tarde) de ambulatório por semana. Nos últimos anos, multiplicaram-se os ambulatórios de subespecialidades, concorrendo para isso a diferenciação do pessoal de cada divisão bem como a mudança da clientela do HCRP. Por exemplo, a Divisão de Cardiologia mantém um ambulatório de Cardiologia Geral e 9 outros ambulatórios. Essa tendência é congruente com o papel reservado ao HCRP no Sistema Único de Saúde. O número de consultas, procedimentos e curativos, realizados nos ambulatórios do DCM, em 2001, foi de 116932.

Na U.E., o DCM participa da responsabilidade pelos atendimentos iniciais, contando para isso com uma equipe fixa de médicos assistentes, no período diurno, e equipes de plantão, constituídas por médico assistente, docente e residentes, no período noturno. No ano de 2001, essas equipes realizaram 8365 atendimentos.

Docentes do DCM são responsáveis por 10 laboratórios especializados do HCRP (Quadro 7). Esses laboratórios atendem a demandas do HCRP e, muitas vezes, servem para fins de pesquisa.

\begin{tabular}{|ccc|}
\hline $\begin{array}{l}\text { Quadro } 6 \text { - Número de leitos e de internações, no } \\
\text { HC-CAMPUS e na U.E., sob responsabilidade do } \\
\text { Departamento de Clínica médica em 2001 }\end{array}$ \\
\hline \multicolumn{4}{c}{ HC-Campus } & U.E. \\
\hline Leitos & 162 & 36 \\
\hline Internaçỗes & 4296 & 3745 \\
\hline
\end{tabular}


Quadro 7 - Laboratórios especializados do HCRP coordenados por docentes do Departamento de Clínica Médica

Dermatologia
Endocrinologia
Gastroenterologia
Hematologia
Imunologia
Nefrologia
Nutriçẫo
Screening
Sorologia
Virologia

São vinculados à Divisões do DCM alguns serviços de apoio médico, onde se realizam exames complementares e procedimentos indispensáveis a uma unidade hospitalar de nível terciário. São eles as seções de Pneumologia e de Cardiologia, o Serviço de Radiodiagnóstico e o setor de Medicina Nuclear.

O Serviço de Radiologia, vinculado à Divisão de Radiologia, além de dar conta da demanda do HCRP, atende às necessidades da rede quanto a exames de tomografia computadorizada e de ressonância magnética.

Somente 2 anos depois de sua criação, quando HCRP foi inaugurado, em 1956, o DCM pode dispor de uma base hospitalar própria. Dez anos depois,em 1966, o DCM contava com 67 leitos em que foram realizadas 835 internações. Nesse mesmo ano, foram atendidas 10632 consultas nos ambulatórios do $D C M$, que eram os seguintes: Geral (todas as manhãs, de segunda a sexta), Cardiologia (quartas à tarde) e Endocrinologia e Nefrologia (sextas à tarde).

\section{PESQUISA}

$O$ encargo da instalação do Departamento de Clínica Médica incluiu o compromisso de conduzir uma experiência nova para o país, a de incorporar a investigação científica ao cotidiano dos docentes clínicos. Para tanto, con- siderou-se essencial a dedicação integral à docência e à pesquisa, como regime de trabalho.

A pesquisa é valorizada no âmbito do DCM. Embora existam diferenças entre as divisões do DCM quanto à produção científica, a atividade de investigação é inerente ao dia-a-dia de todas elas. O resultado é uma volumosa produção de publicações em periódicos nacionais e estrangeiros, de apresentação em eventos científicos, capítulos de livros, relatórios, etc.

Como se pode observar, na Figura 2, a produção de artigos completos do DCM cresceu continuamente, notando-se que o crescimento mais recente, expressivo, se deu sem um aumento contemporâneo do número de docentes, o que indica maior produtividade do sistema. Vale também observar que o aumento mais recente da produção científica coincide com o incremento das responsabilidades do DCM com atividades assistenciais, no âmbito do HCFMRP .

Outro indicador do compromisso do departamento com a atividade científica é o volume de auxílios para pesquisa obtido pelo DCM junto a fontes externas à USP. No biênio 2001-2002, foram aprovados pela FAPESP os relatórios finais de 19 projetos executados por 15 diferentes docentes do DCM, do que resultou a incorporação de bens no valor total de $\mathrm{R} \$ 2.191 .745,50$ ao patrimônio do Departamento. Além do número expressivo de auxílios a projetos individuais, vale lembrar que docentes do DCM os coordenadores de um PRONEX e de um CEPID.

O DCM oferece a alunos do curso de graduação amplas oportunidades de participação em projetos de pesquisa. $\mathrm{O}$ fato é atestado pelos 17 painéis apresentados na Exposição de trabalhos de monitores do DCM, realizada em março de 2002.

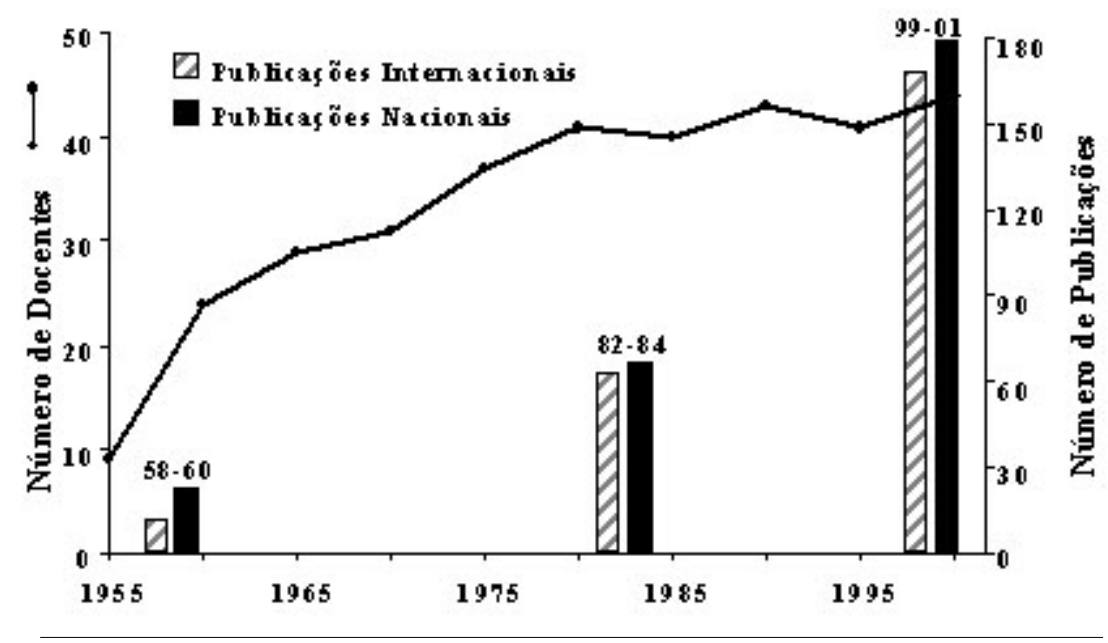

Figura 2: Departamento de Clínica Médica- Evolução do corpo 